УДК 536.24

\title{
НОВЫЙ ПОДХОД К МОДЕЛИРОВАНИЮ ПРОЦЕССА ФОРМИРОВАНИЯ ТЕПЛОВОГО РЕЖИМА ТЕРМОСИФОНОВ БОЛЬШИХ РАЗМЕРОВ ДЛЯ ИСПОЛЬЗОВАНИЯ ГЕОТЕРМАЛЬНОЙ ТЕПЛОТЫ
}

\author{
Максимов Вячеслав Иванович, \\ elf@tpu.ru. \\ Нурпейис Атлант Едилулы, \\ nurpeiis_atlant@mail.ru \\ 1 Национальный исследовательский Томский политехнический университет, \\ Россия, 634050, г. Томск, пр. Ленина, 30.
}

Актуальность исследования обоснована необходимостью разработки математических моделей теплофизических процессов, протекающих в термосифонах, существенно менее сложных, по сравнению с известными (в которых решаются сложные задачи гидродинамики для парового канала), но в то же время обеспечивающих возможность адекватного прогностического моделирования процессов теплопереноса в термосифонах и определения их основных характеристик (температур, тепловых потоков, скоростей испарения), необходимых для создания систем теплоснабжения с использованием геотермальной и петротермальной энергии глубинных слоев земли при передаче теплоты системой термосифонов большой высоты.

Цель: апробация нового подхода к описанию процессов теплопереноса в термосифонах, являющихся основными элементами системы извлечения теплоты глубинных слоев земли (геотермальной и петротермальной энергии) путем сравнения результатов математического моделирования температур в рамках новой модели в характерных точках слоя теплоносителя и результатов экспериментальных исследований.

Объект: замкнутый двухфазный термосифон.

Метод. Краевая задача математической физики решалась методом конечных разностей.

Результаты. На основании анализа и обобщения результатов экспериментальных исследований разработан новый подход к математическому моделированию процесса формирования теплового режима термосифонов большой высоты для использования геотермальной теплоты. Сформулирована математическая модель, описывающая теплоперенос в слое теплоносителя на нижней крышке термосифона и обеспечивающая возможность достоверного прогноза скоростей испарения (или кипения) теплоносителя. Модель отличается от известных описанием не только теплопроводности, но и процесса естественной конвекции в слое теплоносителя. Численные исследования выполнены на пространственной сетке 36×101, шаг по времени изменялся в диапазоне от $10^{-3}$ до $10^{-6} \mathrm{C}$. Рассматривался диапазон тепловых потоков q, соответствующих условиям интенсивного испарения на свободной поверхности слоя теплоносителя. Проведено сравнение температур в точке, расположенной на оси симметрии термосифона на расстоянии 6 мм от поверхности его нижней крышки, полученных при проведении численного анализа и установленных в экспериментах. В качестве теплоносителя рассматривался Н-пентан - низкокипящая жидкость, которая может использоваться в термосифонах при относительно низкой (до $40^{\circ} \mathrm{C}$ ) температуре скальных пород или воды. Установлено хорошее соответствие результатов численного моделирования температурных полей в области анализа и экспериментов. Установлено, что свободная конвекция в слое теплоносителя при достаточно высоких тепловых потоках к нижней поверхности термосифона играет важную роль в формировании температурного поля жидкости и скорости ее испарения со свободной поверхности. Разработанный подход может быть использован при анализе систем геотермального и петротермального теплоснабжения при извлечении теплоты из глубинных слоев земли с использованием группы термосифонов большой высоты.

\section{Ключевые слова:}

Двухфазный термосифон, математическое моделирование, тепловой поток, теплоперенос, испарение, конденсация, термогравитационная конвекция.

\section{Введение}

Одним из перспективных (возможно, даже самым перспективным) направлением в создании систем теплоснабжения будущего является использование геотермальной и петротермальной энергии $[1,2]$. Но ее эффективное использование возможно только при извлечении с больших глубин (до 1 км), где температура среды (грунта, скальных пород) превышает $100{ }^{\circ} \mathrm{C}$. Одним из наиболее реальных способов такого использования теплоты глубинных слоев земли является система из нескольких десятков замкнутых двухфазных термосифонов большой (10-15 м) высоты. Разработка такой системы возможна по результатам комплекса как экспериментальных, так и теоретических исследований. Последние в связи с большими характерными размерами областей исследования, скорее всего, на начальном этапе работы должны быть основными. Но анализ современного состояния теории и эксперимента процессов, протекающих в термосифонах, показывает, что несмотря на достаточно большое число публикаций с результатами экспериментальных, например [3-5], и теоретических, например [6-8], исследований, хорошо известные математические модели (наиболее значимые [9-11]) очень сложны в эксплуатации необходимы большие затраты времени вычислений на анализ одного варианта условий работы термосифонов. Поэтому актуальной является задача разработки математических моделей теплофизических процессов, протекающих в термосифонах, существенно менее сложных, по сравнению, например, с [12-14], но в то же время обеспечивающих возможность адекватного прогностического моделирования процессов теплопереноса в термосифонах и определения их основных харак- 
теристик (температур, тепловых потоков, скоростей испарения).

Анализ и обобщение результатов выполненных ранее экспериментов $[15,16]$ показал, что характерные времена гидродинамических процессов в паровом канале термосифонов (движение пара, в первую очередь) много меньше характерных времен теплопереноса в слое теплоносителя на нижней крышке этого теплообменника. Определяющей характеристикой комплекса процессов, протекающих в термосифоне, является скорость испарения теплоносителя со свободной поверхности его слоя в нижней части парового канала $\left(W_{\text {исп }}\right)$. Вычисление этой величины является во многих случаях достаточно сложной самостоятельной задачей, но в последние годы предложены методы расчета $W_{\text {исп }}$ условиях высоких температур поверхности испарения $[17,18]$, обеспечивающие хорошее соответствие результатов вычислений $W_{\text {исп }}$ и экспериментальных данных даже в условиях высоких температур среды, в которой происходит испарение жидкости.

По результатам экспериментов $[15,16]$ разработан новый подход к описанию теплофизических процессов в термосифоне, отличающийся от известных моделированием теплопереноса только в слое теплоносителя на нижней крышке термосифона, и вычисление по результатам этого моделирования скорости испарения хладагента (основой характеристики работы термосифона).

Цель настоящей работы - апробация этого нового подхода путем сравнения результатов математического моделирования температур в рамках новой модели в характерных точках слоя теплоносителя и результатов экспериментальных исследований.

\section{Постановка задачи}

При постановке задачи использовалась разработанная авторами статьи физическая модель, основой которой является положение о том, что тепловой режим термосифона в полной мере определяется основной характеристикой комплекса взаимосвязанных теплофизических и гидродинамических процессов, протекающих во всех характерных областях этого теплообменника (зоны испарения и конденсации, парового канала, пленки стекающего по вертикальным стенкам термосифона теплоносителя, верхней и нижней крышек, вертикальных стенок), - массовой скорости испарения теплоносителя с поверхности слоя последнего на нижней крышке. При таком подходе принимается, что величина $W_{\text {исп }}$ зависит только от интенсивности теплопереноса в нижней части парового канала.

Задача теплопереноса в этом слое рассматривается в рамках модели, учитывающей механизмы теплопроводности и термогравитационной конвекции. Принято допущение (которое можно квалифицировать как основное), что граница раздела «теплоноситель-пар» не смещается в пространстве (т. е. предполагается, что весь конденсат, образующийся на верхней крышке, успевает возвратиться в зону испарения и компенсировать убыль массы теплоносителя в результате испарения). При этом на основании результатов экспериментальных исследований [19] предполагается, что температура «оборотного» конденсата ниже температуры поверхности испарения. Последнее допущение не является вполне очевидным для условий стекания конденсата по вертикальным стенкам термосифона. Но классическая схема работы термосифонов малой высоты [20], в которой конденсат медленно стекает (в зону испарения) и достаточно интенсивно при этом нагревается потоком высокотемпературных паров, движущихся вверх (в зону конденсации), скорее всего не будет эффективна в термосифонах большой высоты. В последних целесообразно возврат конденсата в зону испарения проводить не в результате пленочного течения по стенкам термосифона, а при падении капель относительно «холодного конденсата» с верхней крышки через паровой канал большой протяженности (до 15 м). В этом случае характерные времена движения капель будут много меньше (в десятки раз) характерного времени стекания пленки. Кроме того, в этом случае температура капель конденсата будет незначительно отличаться от температуры, при которой происходит конденсация на верхней крышке. В результате конденсат возвращается в область испарения очень быстро и с достаточно низкой температурой.

В дополнение обоснованию этого допущения можно отметить, что встречный поток пара будет препятствовать движению капель, но это торможение, как показали эксперименты [21], приведет к тому, что идущая за первой капля догонит ее и сольется с ней. В результате масса капли и, соответственно, действующая на нее сила тяжести увеличиваются. При этом увеличится и скорость падения капли. Процесс движения капель в этих условиях будет самоподдерживающим, как следует из выводов [21]. Кроме того, при падении с высоты нескольких метров скорость капель будет достигать больших значений и следующие во втором, в третьем и других «эшелонах» по высоте парового канала капли будут поглощать идущие впереди. Увеличению размеров капель конденсата, движущихся через паровой канал, также будет способствовать установленный в экспериментах [22, 23] процесс непрерывной трансформации (изменения формы) капель в полете под действием силы тяжести. Установлено $[22,23]$, что капли четырех исследованных типичных жидкостей (скорее всего, это закономерно для ньютоновских жидкостей) последовательно принимают форму сферы; эллипсоида, вытянутого по направлению движения; блина; эллипсоида, вытянутого в поперечном направлении, и ряд других промежуточных форм. В результате поперечные размеры каждой капли циклически увеличиваются почти в два раза [22, 23], и вероятность коагуляции (слияния) движущихся 
в непосредственной близости капель существенно растет. Все эти процессы на практике приведут к тому, что капельный поток будет быстро проходить по паровому каналу из зоны конденсации в зону испарения.

В такой постановке математическая модель процессов теплопереноса в слое конденсата представляет собой систему нестационарных уравнений в частных производных с соответствующими краевыми условиями. Задача решена в осесимметричной постановке. С целью повышения уровня возможных обобщений результатов математического моделирования система уравнений математической физики и соответствующие ей краевые условия записаны в безразмерных переменных.

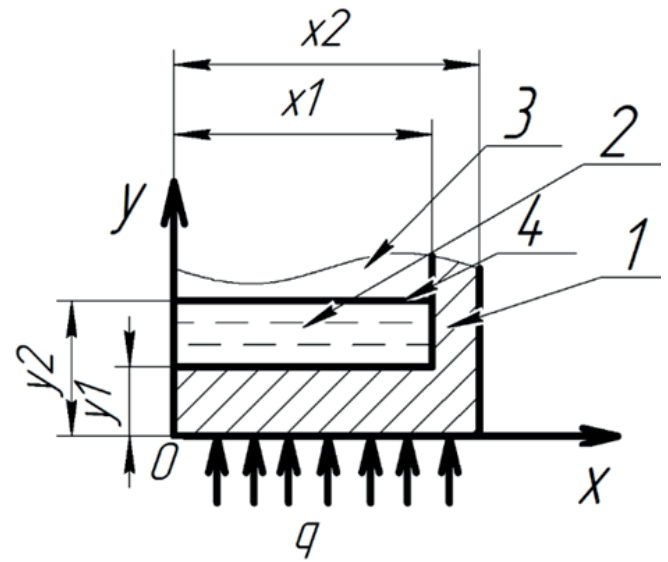

Pис.1. Область решения: 1 - металлический корпус; 2 - слои конденсата; 3 - паровой канал; 4 - поверхность испаре ния

Fig. 1. Area of solution: 1 is the metal case; 2 are the layers of con densate; 3 is the vapor channel; 4 is the surface evaporation

Безразмерные уравнения переноса вихря, Пуассона и энергии в условиях теплопроводности и естественной конвекции в слое теплоносителя и уравнение теплопроводности для стенок термосифона имеют вид [10]:

$$
\begin{gathered}
\frac{\partial \Omega}{\partial \tau}+U \frac{\partial \Omega}{\partial X}+V \frac{\partial \Omega}{\partial Y}=\sqrt{\frac{\operatorname{Pr}}{\operatorname{Ra}}\left(\frac{\partial^{2} \Omega}{\partial X^{2}}+\frac{\partial^{2} \Omega}{\partial Y^{2}}\right)+\frac{\partial \Theta_{2}}{\partial X},} \\
\frac{\partial^{2} \Psi}{\partial X^{2}}+\frac{\partial^{2} \Psi}{\partial Y^{2}}=-\Omega, \\
\frac{\partial \Theta_{2}}{\partial \tau}+U \frac{\partial \Theta_{2}}{\partial X}+V \frac{\partial \Theta_{2}}{\partial Y}=\frac{1}{\sqrt{\operatorname{Ra} \operatorname{Pr}}}\left(\frac{\partial^{2} \Theta_{2}}{\partial X^{2}}+\frac{\partial^{2} \Theta_{2}}{\partial Y^{2}}\right), \\
\frac{1}{\mathrm{Fo}_{1}} \frac{\partial \Theta_{1}}{\partial \tau}=\frac{\partial^{2} \Theta_{1}}{\partial X^{2}}+\frac{\partial^{2} \Theta_{1}}{\partial Y^{2}} .
\end{gathered}
$$

где $\operatorname{Pr}=\frac{v}{a_{2}}-$ число Прандтля; Ra $=\frac{g_{y} \beta\left(T_{h}-T_{0}\right) H^{3}}{1 a_{2}}-$ число Рэлея; $\mathrm{Fo}_{1}=\frac{a_{1} t_{0}}{H^{2}}-$ число Фурье; $X, Y-$ безразмерные координаты декартовой системы коор- динат; $a$-коэффициент температуропроводности; $H=y_{2}$ - характерный размер, м; $t_{0}$ - масштаб времени, с; $\tau$ - безразмерное время; $U, V$ - безразмерные скорости, соответствующие $u, \mathrm{v} ; V_{i n}$ - масштаб скорости, м/c; $T_{0}$ - температура теплоносителя на нижней крышке в начальный момент времени, К; $T_{h}$ - температура кипения хладагента, $\mathrm{K} ; \Theta$ - безразмерная температура; $\psi$ - функция тока, $\mathrm{M}^{2} / \mathrm{c}$; $\psi_{0}$ - масштаб функции тока, $\mathrm{M}^{2} / \mathrm{c} ; \Psi$ - безразмерный аналог $\psi ; \omega$ - завихренность, $1 / \mathrm{c} ; \omega_{0}$ - масштаб завихренности, $1 / \mathrm{c} ; \Omega$ - безразмерный аналог $\omega$.

Начальные условия для уравнений (1)-(4):

$$
\begin{aligned}
\Psi(X, Y, 0) & =\Omega(X, Y, 0)=0, \\
\Theta_{1}(X, Y, 0) & =\Theta_{2}(X, Y, 0)=0,
\end{aligned}
$$

Граничные условия для уравнений (1)-(4):

$$
\begin{aligned}
& X=0, \quad 0 \leq Y \leq Y_{1}: \frac{\partial \Theta_{1}}{\partial X}=0, \\
& X=0, Y_{1} \leq Y \leq Y_{2}: \frac{\partial \Theta_{2}}{\partial X}=0, \quad\left\{\frac{\partial^{2} \Psi_{2}}{\partial X^{2}}=0,\right. \\
& X=X_{2}, \quad 0 \leq Y \leq Y_{2}: \frac{\partial \Theta_{1}}{\partial X}=0, \\
& Y=0, \quad 0 \leq X \leq X_{2}:-\frac{\partial \Theta_{1}}{\partial Y}=K i, \\
& Y=Y_{2}, \quad 0 \leq X \leq X_{1}: \frac{\partial \Theta_{2}}{\partial Y}=\frac{Q_{\dot{e}} W_{\grave{e}} H}{\lambda\left(T_{h}-T_{0}\right)}, \\
& Y=Y_{2}, \quad X_{1} \leq X \leq X_{2}: \frac{\partial \Theta_{1}}{\partial Y}=0, \\
& X=X_{1}, \\
& Y_{1} \leq Y \leq Y_{2}:\left\{\begin{array} { l } 
{ \Theta _ { 1 } = \Theta _ { 2 } , } \\
{ \lambda _ { 1 } \frac { \partial \Theta _ { 1 } } { \partial X } = \lambda _ { 2 } \frac { \partial \Theta _ { 2 } } { \partial X } , }
\end{array} \left\{\begin{array}{l}
\Psi_{2}=0, \\
\frac{\partial \Psi_{2}}{\partial X}=0,
\end{array}\right.\right. \\
& Y=Y_{1}, \\
& 0 \leq X \leq X_{1}:\left\{\begin{array} { l } 
{ \Theta _ { 1 } = \Theta _ { 2 } , } \\
{ \lambda _ { 1 } \frac { \partial \Theta _ { 1 } } { \partial Y } = \lambda _ { 2 } \frac { \partial \Theta _ { 2 } } { \partial Y } , }
\end{array} \left\{\begin{array}{l}
\Psi_{2}=0, \\
\frac{\partial \Psi_{2}}{\partial Y}=0,
\end{array}\right.\right. \\
& W_{e}=\frac{A\left(P_{v s}-P_{v}\right)}{\sqrt{\frac{2 \pi R T}{M}}}, \\
& P_{v s}=P_{0} \exp \left(-\frac{Q_{e} M}{R T}\right), P_{v}=\frac{\rho R T}{M} .
\end{aligned}
$$

Сформулированная краевая задача (1)-(15) решена методом конечных разностей с использованием алгоритма $[24,25]$, разработанного для решения аналогичных задач термогравитационной конвекции в условиях неоднородных граничных 
условий и фазовых переходов на границах раздела. Численные исследования выполнены на пространственной сетке $36 \times 101$, шаг по времени изменялся в диапазоне от $10^{-3}$ до $10^{-6}$ с. Область моделирования приведена на рис. 1. Рассматривался диапазон тепловых потоков $q$, соответствующих условиям интенсивного испарения на свободной поверхности слоя теплоносителя. Режим кипения не рассматривался по двум основным причинам. Первая - условия кипения теплоносителя на нижней крышке термосифона при использовании последовательно каскада последних не достигаются во всех вышерасположенных термосифонах кроме первого, которых при большой глубине размещения каскада может быть несколько десятков. Вторая - кипение приводит к формированию двухфазной среды в слое теплоносителя и турбулизации течения. В этих условиях задача становится чрезвычайно сложной.

На рис. 2 приведены результаты сравнения температур, полученных при проведении численного анализа и установленных в экспериментах $[15,16]$, в точке, расположенной на оси симметрии термосифона на расстоянии 6 мм от поверхности его нижней крышки. В качестве теплоносителя рассматривался Н-пентан - низкокипящая жидкость, которая может использоваться в термосифонах при относительно низкой (до $40{ }^{\circ} \mathrm{C}$ ) температуре скальных пород или воды (такие температуры достигаются на относительно небольших глубинах). Вертикальными короткими линиями обозначены доверительные интервалы определения температур в экспериментах $[15,16]$. Хорошо видно, что теоретический тренд $T(\tau)$ проходит несколько выше (на 0,5 K) экспериментального, но отклонения значений температур в каждый момент времени практически не выходят за границы доверительного интервала определения $T(\tau)$ при доверительной вероятности 0,95 . Можно сделать вывод, что сформулированная в рамках разработанного авторами статьи нового подхода математическая модель теплопереноса в слое теплоносителя на нижней крышке термосифона хорошо соответствует реальному изучаемому процессу при достаточно типичных условия работы таких теплообменников. Можно отметить, что в интервале $\tau$ от 0 до 3500 с температура в контролируемой точке растет, но это увеличение незначительно (менее $3 \mathrm{~K}$ ).

На рис. 3, 4 приведены распределения температур по направлениям осей $x$ и $y$ при $q=0,4 \mathrm{\kappa BT} / \mathrm{m}^{2}$ в точках $y=6$ мм и $x=0$ мм. Видно, что максимальный перепад температур по толщине слоя не превышает 3 K, т. е. процесс термогравитационной конвекции, инициированный подводом теплоты к нижней крышке, приводит к интенсивному перемешиванию жидкости. Перепад температур по поперечной координате также не превышает 3 К при времени 300 с (а-в) и 1 К при 6000 с (г-e), что иллюстрирует интенсивный процесс выравнивания температур и по поперечной координате.

На рис. 5 приведены линии тока в слое теплоносителя на нижней крышке термосифона при $q=0,4 \mathrm{\kappa Bт} / \mathrm{M}^{2}(\tau=300$ с и $\tau=6000 \mathrm{c})$. Видно, что в правой половине слоя теплоносителя формируется одна достаточно интенсивная вихревая структура (скорости в продольном и поперечном направлениях достигают 0,5 мм/с при температурах среды от 303 до 309 К). С ростом времени скорости естественной конвекции растут вследствие подъема температуры нижней крышки термосифона и, соответственно, теплоносителя в нижней части слоя. Можно сделать вывод, что термогравитационная конвекция является основным механизмом формирования температурного поля слоя теплоносителя на нижней крышке термосифона и, соответственно, температуры свободной поверхности, величина которой в рассматриваемых условиях определяет скорость испарения теплоносителя.

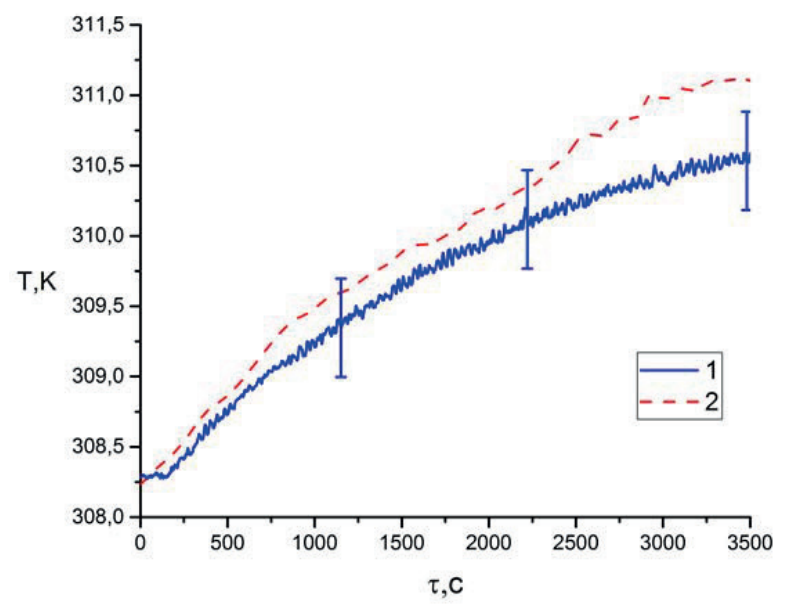

$\sigma / b$

Pис. 2. Телпературные зависимости в точке $x=0$ мл, $y=6$ мл от врелени для слоя теплоносителя (Н-пентан), а) q=0,4 кВт/л", б) $q=0,5 \kappa \mathrm{Bm} / \mathrm{m}^{2},(1$ - эксперимент, 2 - численное моделирование (- - ))

Fig. 2. Temperature dependencies in the point $x=0 \mathrm{~mm}, y=6 \mathrm{~mm}$ on time for the coolant layer (n-pentane), a) $\left.q=0,4 \mathrm{~kW} / \mathrm{m}^{2}, b\right) q=0,5 \mathrm{~kW} / \mathrm{m}^{2}$, (1-experiment, 2 - numerical modeling (--)) 


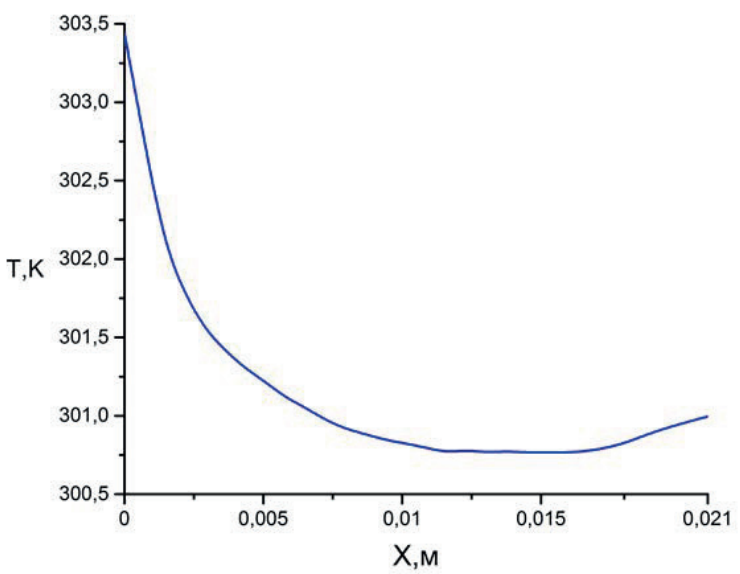

$a / a$

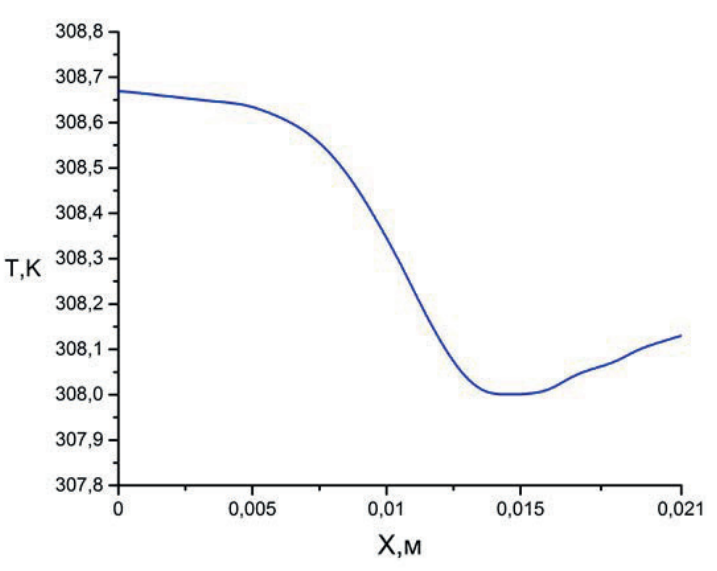

$\sigma / b$

Pис.3. Распределения телператур по оси $X$ в сечении $Y=6$ мм в слое теплоносителя на нижней крышке терлосифона при $q=0,4 \kappa B m / m^{2}$ и времени: а) $300 c$, б) $6000 c$

Fig. 3. Temperature distribution along the X-axis in the cross-section $Y=6 \mathrm{~mm}$ in the coolant layer on the bottom cover of thermosiphon at $q=0,4 \mathrm{~kW} / \mathrm{m}^{2}$ and time: a) $\left.300 \mathrm{~s} ; \mathrm{b}\right) 6000 \mathrm{~s}$
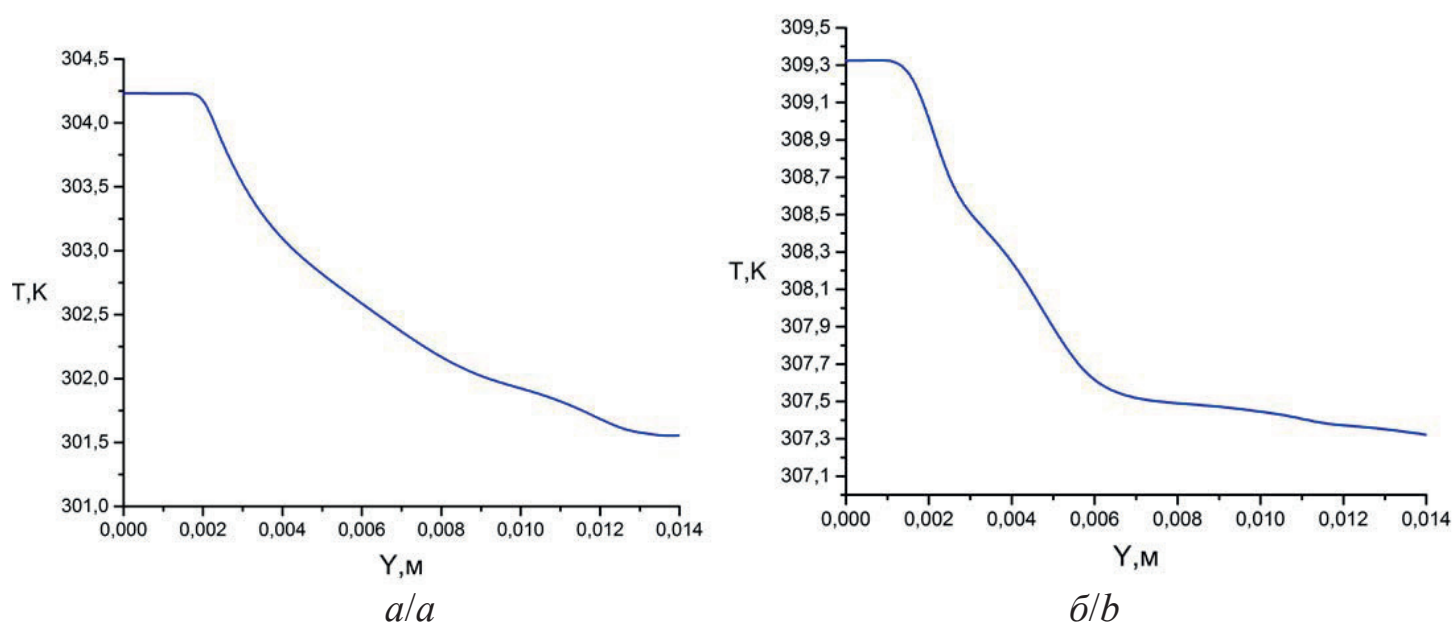

Рис.4. Распределения температур по оси $Y$ в сечении $Y=6$ мл в слое теплоносителя на нижней крышке терлосифона при $q=0,4 \mathrm{\kappa Bm} / \mathrm{m}^{2}$ и времени: а) $300 \mathrm{c}$, б) $6000 \mathrm{c}$

Fig. 4. Temperature distribution along the $Y$-axis in the cross-section $Y=6 \mathrm{~mm}$ in the coolant layer on the bottom cover of thermosiphon at $q=0,4 \mathrm{~kW} / \mathrm{m}^{2}$ and time: a) $\left.300 \mathrm{~s} ; b\right) 6000 \mathrm{~s}$
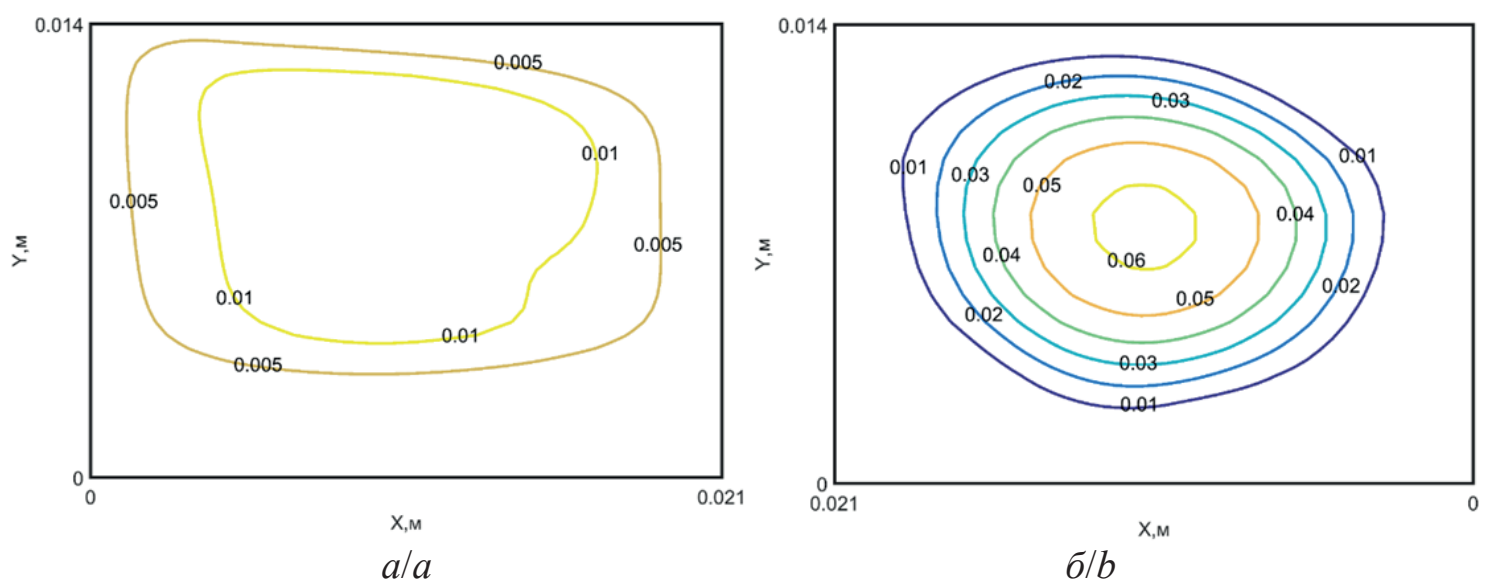

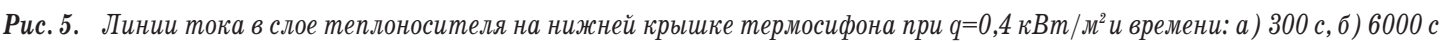

Fig. 5. Current lines in the coolant layer on the bottom cover of thermosiphon at $q=0,4 \mathrm{~kW} / \mathrm{m}^{2}$ and time: a) $\left.300 \mathrm{~s} ; b\right) 6000 \mathrm{~s}$ 


\section{Заключение}

По результатам анализа и обобщения выполненных ранее экспериментальных исследований сформирована математическая модель теплопереноса на нижней крышке термосифона, отличающаяся от известных учетом процессов термогравитационной конвекции. Сравнение результатов численного моделирования с использованием этой модели и полученных в экспериментах зависимостей температур в характерных точках области анализа от времени показало их хорошее соответствие. Установлено, что свободная конвекция в слое теплоносителя при достаточно высоких те-

\section{СПИСОК ЛИТЕРАТУРЫ}

1. Томаров Г.В., Шипков А.А. Современная геотермальная энергетика: геотермальные электростанции с бинарным циклом // Теплоэнергетика. - 2017. - № 4. - С. 3-12.

2. Гнатусь Н.А., Хуторской М.Д., Хмелевской В.К. Петротермальная геоэнергетика и геофизика // Вест. Моск. Ун-та. Сер. 4. Геология. - 2011. - № 3. - С. 3-9.

3. Kim C., Lee K.-S., Yook S.-J. Effect of air-gap fans on cooling of windings in a large-capacity, high-speed induction motor // Applied Thermal Engineering. - 2016. - V. 100. - P. 658-667.

4. Thermal enhancement by using grooves and ribs combined with delta-winglet vortex generator in a solar receiver heat exchanger / L. Luo, F. Wen, L., Wang B. Sundén, S. Wang // Applied Energy. - 2016. - V. 183. - P. 1317-1332.

5. Ibrahim E., Moawed M., Berbish N.S. Heat transfer characteristics of rotating triangular thermosyphon // Heat Mass Transfer. - 2012. - V. 48. - P. 1539-1548.

6. Two-phase closed thermosyphons: a review of studies and solar applications / D. Jafari, A. Franco, S. Filippeschi, P. Di Marco // Renewable and Sustainable Energy Reviews. - 2016. - V. 53. P. 575-593.

7. Theoretical and Experimental Analysis of the Steady Flow Across the Cylinderhead of a Low-Capacity Engine / A. Castilla, M. Rubio, C. Ferrera, J.M. Montanero, J. Fernández // Journal of Applied Mechanics, Transactions ASME. - 2016. - V. 83. - Iss. 12. 4 p. DOI: $10.1115 / 1.4034619$.

8. Kumar A., Dhiman A., Baranyi L. Fluid flow and heat transfer around a confined semi-circular cylinder: Onset of vortex shedding and effects of Reynolds and Prandtl numbers // International Journal of Heat and Mass Transfer. - 2016. - V. 102. P. $417-425$.

9. Kuznetsov G.V., Sitnikov A.E. Numerical analysis of basic regularities of heat and mass transfer in high-temperature heat pipe // TVT. - 2002. - V. 40. - Iss. 6. - P. 964-970.

10. Kuznetsov G.V., Al-Ani M.A., Sheremet M.A. Numerical analyses of convective heat transfer in a closed two-phase thermosiphon // Journal of Engineering Thermophysics. - 2011. - V. 20 (2). P. 201-210.

11. Fadhl B., Wrobel L.C., Jouhara H. Numerical modelling of the temperature distribution in a two-phase closed thermosyphon // Applied Thermal Engineering. - 2013. - V. 60 - P. 122-131.

12. Gautam P., Chandy A.J. Numerical investigation of the air pumping noise generation mechanism in tire grooves // Journal of Vibration and Acoustics, Transactions of the ASME. - 2016. V. 135 . - Iss. $5 .-8$ p. DOI: $10.1115 / 1.4033342$.

13. Numerical modeling of current loads on a net cage considering fluid-structure interaction / Y. Yao, Y. Chen, H. Zhou, H. Yang // Journal of Fluids and Structures. - 2016. - V. 62. - P. 350-366.

14. Gilani S., Montazeri H., Blocken B. CFD simulation of stratified indoor environment in displacement ventilation: Validation and пловых потоках к нижней поверхности термосифона играет важную роль в формировании температурного поля жидкости и скорости ее испарения со свободной поверхности. Результаты численных исследований дают основания для вывода о перспективности использования предложенного в работе подхода к описанию тепловых режимов замкнутых двухфазных термосифонов.

Исследование проведено в рамках програмлы повышения конкурентоспособности Национального исследовательского Томского политехнического университета среди ведущих мировых научно-образовательных иентров (Госзадание "Наука» 8.13264.2018/8.9, проект ВИУИШЭ-300/2018).

sensitivity analysis // Building and Environment. - 2016. V. 95. - P. 299-313.

15. Nurpeiis A., Ponomarev K., Nemova T. Peculiarities of temperature fields formation in vapor channels of thermosyphons with heat carriers boiling at low temperatures // MATEC Web of Conf. - 2017. - V. 141. - 4 p. URL: doi.org/10.1051/matecconf/201714101005 (дата обращения 29.03.2019).

16. Nurpeiis A., Orlova E., Ponomarev K. An experimental study of the influence of a thermosyphon filling ratio on a temperature distribution in characteristic points along the vapor channel height // MATEC Web of Conf. - 2017. - V. 110. - 5 p. URL: doi.org/10.1051/matecconf/201711001062 (дата обращения 29.03.2019).

17. Kuznetsov G.V., Strizhak P.A. Evaporation of single droplets and dispersed liquid flow in motion through high-temperature combustion products // High Temperature. - 2014. - V. 52. P. 568-575.

18. Kuznetsov G.V., Strizhak P.A. Numerical investigation of the influence of convection in a mixture of combustion products on the integral characteristics of the evaporation of a finely atomized water drop // Journal of Engineering Physics and Thermophysics. - 2014. - V. 87. - P. 103-111.

19. Fadhl B., Wrobel L.C., Jouhara H. Numerical modelling of the temperature distribution in a two-phase closed thermosyphon // Applied Thermal Engineering. - 2013. - V. 60. - P. 122-131.

20. Eidan A.A., Najim S.E., Jalil J.M. An experimental and a numerical investigation of HVAC system using thermosyphon heat exchangers for sub-tropical climates // Applied Thermal Engineering. - 2017. - V. 114. - P. 693-703.

21. Volkov R.S., Kuznetsov G.V., Strizhak P.A. Evaporation of two liquid droplets moving sequentially through high-temperature combustion products // Thermophysics and Aeromechanics. April 2014. - V 21. - Iss. 2. - P. 255-258.

22. Kuznetsov G.V., Strizhak P.A. The motion of a manifold of finely dispersed liquid droplets in the counterflow of high-temperature gases // Technical Physics Letters. - July 2014. - V. 40. Iss. 6. - P. 499-502.

23. Volkov R.S., Kuznetsov G.V., Strizhak P.A. Influence of the initial parameters of spray water on its motion through a counter flow of high-temperature gases // Technical Physics. - July 2014. - V. 59. - Iss. 7. - P. 959-967.

24. Самарский А.А., Вабищевич П.Н. Численные методы решения задач конвекции-диффузии. - М.: Эдиториал УРСС, 1999. - 248 с.

25. Mathematical simulation of thermophysical and thermos chemical processes during combustion of intumescent fire-protective coating / V.L. Strakhov, A.N. Garaschenko, G.V. Kuznetsov, V.P. Rudzinskii // Combustion, Explosion and Shock Waves. 2001. - V. 37. - P. 178-186.

Поступила 03.04.2019 2. 


\section{Информация об авторах}

Максимов В.И., кандидат технических наук, доцент Научно-образовательного центра И.Н. Бутакова, Инженерной школы энергетики Национального исследовательского Томского политехнического университета.

Hyрпейис A.E., ассистент Научно-образовательного центра И.Н. Бутакова, Инженерной школы энергетики Национального исследовательского Томского политехнического университета. 


\title{
NEW APPROACH TO MODELLING THE FORMATION OF LARGE-SIZED THERMOSIPHONS THERMAL REGIME FOR USING GEOTHERMAL HEAT
}

\author{
Vyacheslav I. Maksimov', \\ elf@tpu.ru

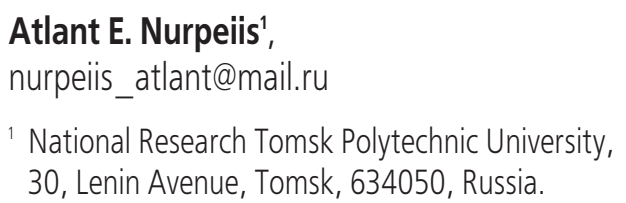

The relevance of the research is caused by the necessity to develop mathematical models of thermophysical processes occurring in thermosiphons. These models are significantly less complex than the known ones, where sophisticated hydrodynamics problems are solved for vapor channel. However, at the same time they provide the possibility of adequate predictive modeling of heat transfer processes in thermosiphons and determining their main characteristics (temperature, heat fluxes, and evaporation rates) which are necessary to create heat supply systems using geothermal and petrothermal energy of the deep layers of the earth when heat transfers by high thermosiphons system.

The main aim of the research is the validation of new approach to description of heat transfer in thermosiphons, which are the main elements of the system for extracting heat from the deeper layers of the earth (geothermal and petrothermal energy) by comparing the results of mathematical modeling of temperatures within the framework of the new model at characteristic points of the coolant layer and experimental results.

Object: two-phase close thermosiphon

Method. The formulated boundary problem of mathematical physics was solved by the finite difference method.

Results. Based on the analysis and synthesis of experimental results, the authors have developed a new approach to mathematical modeling of thermal regime formation of high thermosiphons for using geothermal heat. We formulated mathematical modeling describing heat transfer in a coolant layer on the bottom cover of thermosiphon. This model provides to make reliable prediction of evaporation (or boiling) rates of a coolant. The model differs from the known ones by description of conduction, as well as natural convection in the coolant layer. A good agreement was established between the results of numerical calculations of temperature fields in the area of analysis and the experiments. Numerical studies were performed on a spatial grid of 36×101, the time step was varied in the range from $10^{-3}$ to $10^{-6} \mathrm{~s}$. We considered the range of heat fluxes q corresponding to the conditions of intense evaporation on the free surface of the coolant layer. Experimentally and numerically obtained temperatures at a point located on the symmetry axis of a thermosiphon at a distance of $6 \mathrm{~mm}$ from the surface of its bottom cover were compared. $\mathrm{N}$-pentane, a low-boiling liquid that can be used in thermosiphons at relatively low temperature (up to $40{ }^{\circ} \mathrm{C}$ ) of rock or water, was considered as a coolant. The temperature fields obtained in the experiments and numerical simulations agree well. Natural convection in the coolant layer at sufficiently high heat fluxes to the lower surface of the thermosiphon plays an important role in formation of liquid temperature field and the rate of its evaporation from the free surface. The developed approach can be used for analysis of geothermal and petrothermal heat supply systems when extracting heat from the deeper layers of the earth using a group of high thermosiphons.

\section{Key words:}

Two-phase thermosiphon, mathematical modelling, heat flux, heat transfer, evaporation, condensation, thermo-gravitational convection.

The study was conducted in the framework of the program of increasing the competitiveness of National Research Tomsk Polytechnic University among world's leading research and educational centers (state assignment «Science» 8.13264.2018/8.9, project VIU-ISHE-300/2018).

\section{REFERENCES}

1. Tomarov G.V., Shipkov A.A. Modern geothermal power: Binary cycle geothermal power plants. Thermal Engineering, 2017, no. 4, pp. 3-12. In Rus.

2. Gnatus N.A., Khutorskoy M.D., Khmelevskoy V.K. Petrothermal energy and Geophysics. Moscow University Geology Bulletin. Geology, 2011, no. 3, pp. 3-9. In Rus.

3. Kim C., Lee K.-S., Yook S.-J. Effect of air-gap fans on cooling of windings in a large-capacity, high-speed induction motor. Applied Thermal Engineering, 2016, vol. 100, pp. 658-667.

4. Luo L., Wen F., Wang L., Sundén B., Wang S. Thermal enhancement by using grooves and ribs combined with delta-winglet vortex generator in a solar receiver heat exchanger. Applied Energy, 2016, vol. 183, pp. 1317-1332.

5. Ibrahim E., Moawed M., Berbish N. S. Heat transfer characteristics of rotating triangular thermosiphon. Heat Mass Transfer, 2012, vol. 48, pp. 1539-1548.
6. Jafari D., Franco A., Filippeschi S., Di Marco P. Two-phase closed thermosyphons: a review of studies and solar applications. Renewable and Sustainable Energy Reviews, 2016, vol. 53, pp. 575-593.

7. Castilla A., Rubio M., Ferrera C., Montanero J. M., Fernández J. Theoretical and Experimental Analysis of the Steady Flow Across the Cylinderhead of a Low-Capacity Engine. Journal of Applied Mechanics, Transactions ASME, 2016, vol. 83, Iss. 12, 4 p. DOI: $10.1115 / 1.4034619$.

8. Kumar A., Dhiman A., Baranyi L. Fluid flow and heat transfer around a confined semi-circular cylinder: Onset of vortex shedding and effects of Reynolds and Prandtl numbers. International Journal of Heat and Mass Transfer, 2016, vol. 102, pp. 417-425.

9. Kuznetsov G.V., Sitnikov A.E. Numerical analysis of basic regularities of heat and mass transfer in high-temperature heat pipe. TVT, 2002, vol. 40, Iss. 6, pp. 964-970.

10. Kuznetsov G.V., Al-Ani M.A., Sheremet M.A. Numerical analyses of convective heat transfer in a closed two-phase thermos- 
iphon. Journal of Engineering Thermophysics, 2011, vol. 20 (2), pp. 201-210.

11. Fadhl B., Wrobel L.C., Jouhara H. Numerical modelling of the temperature distribution in a two-phase closed thermosiphon. Applied Thermal Engineering, 2013, vol. 60, pp. 122-131.

12. Gautam P., Chandy A.J. Numerical investigation of the air pumping noise generation mechanism in tire grooves. Journal of $\mathrm{Vi}$ bration and Acoustics, Transactions of the ASME, 2016, vol. 135, Iss. 5,8 p. DOI: $10.1115 / 1.4033342$.

13. Yao Y., Chen Y., Zhou H., Yang H. Numerical modeling of current loads on a net cage considering fluid-structure interaction. Journal of Fluids and Structures, 2016, vol. 62, pp. 350-366.

14. Gilani S., Montazeri H., Blocken B. CFD simulation of stratified indoor environment in displacement ventilation: Validation and sensitivity analysis. Building and Environment, 2016, vol. 95, pp. 299-313.

15. Nurpeiis A., Ponomarev K., Nemova T. Peculiarities of temperature fields formation in vapor channels of thermosyphons with heat carriers boiling at low temperatures. MATEC Web of Conf., 2017. Vol. 141, 4 p. Available at: doi.org/10.1051/matecconf/201714101005 (accessed 29 March 2019).

16. Nurpeiis A., Orlova E., Ponomarev K. An experimental study of the influence of a thermosyphon filling ratio on a temperature distribution in characteristic points along the vapor channel height. MATEC Web of Conf., 2017. Vol. 110, 5 p. Available at: doi.org/10.1051/matecconf/201711001062 (accessed 29 March 2019).

17. Kuznetsov G.V., Strizhak P.A. Evaporation of single droplets and dispersed liquid flow in motion through high-temperature combustion products. High Temperature, 2014, V. 52 , pp. $568-575$.

18. Kuznetsov G.V., Strizhak P.A. Numerical investigation of the influence of convection in a mixture of combustion products on the integral characteristics of the evaporation of a finely atomized water drop. Journal of Engineering Physics and Thermophysics, 2014, vol. 87, pp. 103-111.

19. Fadhl B., Wrobel L.C., Jouhara H. Numerical modelling of the temperature distribution in a two-phase closed thermosiphon. Applied Thermal Engineering, 2013, vol. 60, pp. 122-131.

20. Eidan A.A., Najim S.E., Jalil J.M. An experimental and a numerical investigation of HVAC system using thermosyphon heat exchangers for sub-tropical climates. Applied Thermal Engineering, 2017, vol. 114, pp. 693-703.

21. Volkov R.S. Kuznetsov G.V., Strizhak P.A. Evaporation of two liquid droplets moving sequentially through high-temperature combustion products. Thermophysics and Aeromechanics, April 2014, vol. 21, Iss. 2, pp. 255-258.

22. Kuznetsov G.V., Strizhak P.A. The motion of a manifold of finely dispersed liquid droplets in the counterflow of high-temperature gases. Technical Physics Letters, July 2014, vol. 40, Iss. 6, pp. 499-502.

23. Volkov R.S., Kuznetsov G.V., Strizhak P.A. Influence of the initial parameters of spray water on its motion through a counter flow of high-temperature gases. Technical Physics, July 2014, vol. 59, Iss. 7, pp. 959-967.

24. Samarskiy A.A., Vabishchevich P.N. Chislennye metody resheniya zadach konvektsii-diffuzii [Numerical methods for solving convection-diffusion problems]. Moscow, Editorial URSS Publ., 1999. 248 p. In Rus.

25. Strakhov V.L., Garaschenko A.N., Kuznetsov G.V., Rudzinskii V.P. Mathematical simulation of thermophysical and thermos chemical processes during combustion of intumescent fireprotective coating. Combustion, Explosion and Shock Waves, 2001, vol. 37, pp. 178-186.

Received: 3 April 2019.

\section{Information about the authors}

Vyacheslav I. Maksimov, Cand. Sc., associate professor, National Research Tomsk Polytechnic University.

Atlant E. Nurpeiis, assistant, National Research Tomsk Polytechnic University. 